\title{
Seepage Characteristics Study on Power-Law Fluid in Fractal Porous Media
}

\author{
Meijuan Yun ${ }^{1,2}$ \\ ${ }^{1}$ College of Science, Wuhan University of Science and Technology, Wuhan 430081, China \\ ${ }^{2}$ State Key Laboratory of Petroleum Resources and Prospecting, China University of Petroleum, Beijing 102249, China
}

Correspondence should be addressed to Meijuan Yun; yunmeijuanwei@aliyun.com

Received 27 August 2013; Revised 8 January 2014; Accepted 9 January 2014; Published 23 February 2014

Academic Editor: Mohamed Seddeek

Copyright (C) 2014 Meijuan Yun. This is an open access article distributed under the Creative Commons Attribution License, which permits unrestricted use, distribution, and reproduction in any medium, provided the original work is properly cited.

We present fractal models for the flow rate, velocity, effective viscosity, apparent viscosity, and effective permeability for powerlaw fluid based on the fractal properties of porous media. The proposed expressions realize the quantitative description to the relation between the properties of the power-law fluid and the parameters of the microstructure of the porous media. The model predictions are compared with related data and good agreement between them is found. The analytical expressions will contribute to the revealing of physical principles for the power-law fluid flow in porous media.

\section{Introduction}

The power-law fluid is one of non-Newtonian fluids and its constitutive equation is [1]

$$
\tau=\mu \dot{\gamma}^{n},
$$

where $\tau, \mu, \dot{\gamma}$, and $n$ are shear stress, consistency index, shear rate, and power index, respectively. Typical power-law fluids include some colloids, milk, gelatin, blood, and liquid cement. The power-law fluid model can be reduced to the Newtonian fluid model when $n=1$.

Not all types of porous media can be described by the fractal theory, but it has been shown that porous media in nature such as oil/water reservoirs have the fractal characters [2-17], so the fractal theory may be used to analyze the transport properties of natural porous media. Yu et al. [7, 8] developed a fractal permeability model based on the assumptions that porous media are composed of a set of parallel tortuous capillaries and the porous media naturally formed are fractals. Karacan and Halleck [9] applied Yu and Cheng's model [7] to a set of sandstone porous samples with cylindrical structures and the permeability data predicted by the fractal model are in agreement with their experimental measurements. Xiao et al. [10, 11] have developed some models for heat transfer of fluids by fractal technique. Cai et al. [12-17] developed fractal models based on spontaneous imbibition effect. However, the above fractal models developed are based on the Newtonian fluid flow in porous media and they are not applicable to those of non-Newtonian fluid flow in porous media. This work is devoted to the studying of the flow characteristics of power-law fluid in fractal porous media.

\section{Fractal Theory for Porous Media}

In the present work, it is assumed that the porous medium model is a bundle of tortuous capillary tubes following a fractal behavior, mentioned in the earlier publication of $\mathrm{Yu}$ and Cheng [7], with constant straight distance $L_{0}$.

The porous media in nature can be and have been described as fractal objects, and the size distribution of pores in porous media exhibits the fractal characteristics and that the cumulative number $N$ of pores in porous media whose sizes are greater than or equal to $r$ follows the power law relation $[6-8]$ :

$$
N(L \geq r)=\left(\frac{r_{\max }}{r}\right)^{D_{f}}
$$

where $L$ is measurement scale, $D_{f}$ is the pore area fractal dimension, $0<D_{f}<2$ in two dimensions, $r_{\max }$ is the maximum pore size, and $r$ is the pore sizes. 
According to (2), the total pore number $N_{t}$ from the smallest pore size to the largest pore size can be expressed by [7]

$$
N_{t}\left(L \geq r_{\min }\right)=\left(\frac{r_{\max }}{r_{\min }}\right)^{D_{f}} .
$$

Differentiating (2) with respect to $r$ yields

$$
-d N=D_{f} r_{\max }^{D_{f}} r^{-\left(D_{f}+1\right)} d r .
$$

Dividing (4) by (3) results in

$$
-\frac{d N}{N_{t}}=D_{f} r_{\min }^{D_{f}} r^{-\left(D_{f}+1\right)} d r=f(r) d r,
$$

where $f(r)=D_{f} r_{\min }^{D_{f}} r^{-\left(D_{f}+1\right)}$ is the probability density function. Based on the normalization condition, the probability density function should obey the following normalization relationship:

$$
\int_{r_{\min }}^{r_{\max }} f(r) d r=1-\left(\frac{r_{\min }}{r_{\max }}\right)^{D_{f}}=1 .
$$

Equation (6) holds if and only if

$$
\left(\frac{r_{\min }}{r_{\max }}\right)^{D_{f}} \cong 0
$$

is satisfied. Equation (7) can be considered as a criterion of whether a porous medium can be characterized by fractal theory and technique. In general [7], most porous media have $r_{\min } / r_{\max } \leq 10^{-2}$; thus (7) approximately holds. Thus, the fractal theory and technique can be used to analyze the characters of porous media.

The fractal dimension $D_{f}$ in (2)-(7) for pore space is given by [6-8]

$$
D_{f}=2-\frac{\ln \phi}{\ln \left(r_{\min } / r_{\max }\right)},
$$

where $\phi$ is the porosity of the porous medium.

Because the tortuosity of capillaries has been proven to follow the fractal scaling laws, the total length of a tortuous capillary can be expressed as [6-8]

$$
L_{t}=L_{0}^{D_{T}}(2 r)^{1-D_{T}}
$$

where $L_{t}$ and $L_{0}$ are the actual length and straight distance of a tortuous capillary, respectively, and $D_{T}$ is the tortuosity fractal dimension of tortuous capillaries with $1<D_{T}<3$, meaning the extent of tortuousness of capillary pathways for fluid flow through a porous medium. $D_{T}$ is given by [6]

$$
D_{T}=1+\frac{\ln T}{\ln \left(L_{0} / 2 r_{\mathrm{av}}\right)},
$$

where $T$ is the average tortuosity of tortuous capillaries and $r_{\text {av }}$ is the average radius of capillaries. The purpose of introducing the tortuosity of tortuous capillaries is to include the effect of the complexity of the geometrical shape on fluid permeability. For flow paths in porous media, the relation between the average tortuosity and porosity can be obtained as [6]

$$
\begin{aligned}
T=\frac{1}{2}\left[1+\frac{1}{2} \sqrt{1-\phi}+\sqrt{1-\phi}\right. \\
\\
\left.\times \frac{\sqrt{((1 / \sqrt{1-\phi})-1)^{2}+1 / 4}}{1-\sqrt{1-\phi}}\right] .
\end{aligned}
$$

The average radius $r_{\mathrm{av}}$ of capillaries can be found with the aid of (5)

$$
r_{\mathrm{av}}=\int_{r_{\min }}^{r_{\max }} r f(r) d r=\frac{D_{f} r_{\min }}{D_{f}-1}\left[1-\left(\frac{r_{\min }}{r_{\max }}\right)^{D_{f}-1}\right] .
$$

The structural parameters for fractal porous media are given by [6]

$$
\begin{gathered}
L_{0}=R \sqrt{\frac{2 \pi}{\sqrt{3}(1-\phi)}}, \\
r_{\max }=\frac{R}{2} \sqrt{\frac{2 \phi}{1-\phi}}, \\
\frac{r_{\min }}{r_{\max }}=\frac{\sqrt{2(1-\phi)}}{24},
\end{gathered}
$$

where $R$ is the average particle radius in porous media.

Once the value of the average particle radius $R$ and the porosity $\phi$ are found, the microstructure parameters and the fractal dimensions of porous media can be determined.

Equations (2)-(15) form the theoretical base of the present work, and the fractal models for power-law fluid flow in porous media are derived in the next section.

\section{Fractal Models for Power-Law Fluid Flow in Porous Media}

The flow rate through a tortuous capillary with radius $r$ for power-law fluid is [1]

$$
q(r)=\frac{n \pi r^{3+1 / n} \Delta p^{1 / n}}{(3 n+1)\left(2 \mu L_{t}\right)^{1 / n}}
$$

where $\Delta p$ is the pressure drop.

According to (9), (16) can be modified as

$$
q(r)=\frac{n \pi r^{3+D_{T} / n} \Delta p^{1 / n}}{(3 n+1) 2^{\left(2-D_{T}\right) / n} \mu^{1 / n} L_{0}^{D_{T} / n}} .
$$


The total flow rate $Q$ over the cross section can be obtained by integrating (17) over the entire range of pore sizes

$$
\begin{aligned}
Q= & -\int_{r_{\min }}^{r_{\max }} q(r) d N \\
= & \frac{n \pi D_{f} r_{\max }^{3+D_{T} / n} \Delta p^{1 / n}}{(3 n+1)\left(3+\left(D_{T} / n\right)-D_{f}\right) 2^{\left(2-D_{T}\right) / n} \mu^{1 / n} L_{0}^{D_{T} / n}} \\
& \times\left[1-\left(\frac{r_{\min }}{r_{\max }}\right)^{3+\left(D_{T} / n\right)-D_{f}}\right]
\end{aligned}
$$

since $1<D_{T}<3$ and $0<D_{f}<2$; the exponent $3+\left(D_{T} / n\right)-D_{f}>1$; in general $r_{\min } / r_{\max } \sim 10^{-2}$; thus $\left(r_{\min } / r_{\max }\right)^{3+\left(D_{T} / n\right)-D_{f}} \ll 1$. So (18) can be reduced to

$$
Q=\frac{n \pi D_{f} r_{\max }^{3+D_{T} / n} \Delta p^{1 / n}}{(3 n+1)\left(3+\left(D_{T} / n\right)-D_{f}\right) 2^{\left(2-D_{T}\right) / n} \mu^{1 / n} L_{0}^{D_{T} / n}} .
$$

The total pore volume is given by

$$
\begin{aligned}
v_{p}= & -\int_{r_{\min }}^{r_{\max }} \pi r^{2} L_{t} d N \\
= & \frac{\pi D_{f} L_{0}^{D_{T}} r_{\max }^{3-D_{T}}}{2^{D_{T}-1}\left(3-D_{T}-D_{f}\right)} \\
& \times\left[1-\left(\frac{r_{\min }}{r_{\max }}\right)^{3-D_{T}-D_{f}}\right] .
\end{aligned}
$$

The porosity is

$$
\phi=\frac{v_{p}}{v_{t}}=\frac{v_{p}}{A L_{0}},
$$

where $v_{t}$ is the total volume of porous media.

According to (20) and (21), the cross-sectional area $A$ of porous media is

$$
\begin{aligned}
A= & \frac{\pi D_{f} L_{0}^{D_{T}-1} r_{\max }^{3-D_{T}}}{\phi 2^{D_{T}-1}\left(3-D_{T}-D_{f}\right)} \\
& \times\left[1-\left(\frac{r_{\min }}{r_{\max }}\right)^{3-D_{T}-D_{f}}\right] .
\end{aligned}
$$

Dividing (19) by (22) gives the average flow velocity for power-law fluid in porous media

$$
\begin{aligned}
V=\left(n r_{\max }^{\left(D_{T} / n\right)+D_{T}} \phi 2^{D_{T^{-1}}-\left(\left(2-D_{T}\right) / n\right)}\left(3-D_{T}-D_{f}\right) \Delta p^{1 / n}\right) \\
\times\left((3 n+1)\left(3+\left(\frac{D_{T}}{n}\right)-D_{f}\right)\right. \\
\left.\times \mu^{1 / n} L_{0}^{\left(D_{T} / n\right)+D_{T}-1}\left[1-\left(\frac{r_{\min }}{r_{\max }}\right)^{3-D_{T}-D_{f}}\right]\right)^{-1} .
\end{aligned}
$$

We can see that the flow velocity for power-law fluid in porous media is related not only to the structural parameters of porous media $\left(\phi, D_{f}, D_{T}, L_{0}, r_{\min }\right.$, and $\left.r_{\max }\right)$ and pressure drop $(\Delta p)$ but also to fluid characteristic parameters $(\mu, n)$.

When $n=1$ in (23), the flow velocity for the power-law model reduces to that for the Newtonian model:

$$
V=\frac{r_{\max }^{2 D_{T}} \phi 2^{2 D_{T}-5}\left(3-D_{T}-D_{f}\right) \Delta p}{\left(3+D_{T}-D_{f}\right) \mu L_{0}^{2 D_{T}-1}\left[1-\left(r_{\min } / r_{\max }\right)^{3-D_{T}-D_{f}}\right]} .
$$

According to Darcy law $V=(K / \mu)\left(\Delta p / L_{0}\right)$, we obtain the absolute permeability $K$ of porous media:

$$
K=\frac{r_{\max }^{2 D_{T}} \phi 2^{2 D_{T}-5}\left(3-D_{T}-D_{f}\right)}{\left(3+D_{T}-D_{f}\right) L_{0}^{2 D_{T}-2}\left[1-\left(r_{\min } / r_{\max }\right)^{3-D_{T}-D_{f}}\right]}
$$

The relation between the flow velocity and the pressure gradient for power-law fluid flow in packed beds is given by [18]

$$
\begin{gathered}
V=\left(\frac{K}{\mu_{\mathrm{eff}}} \frac{\Delta p}{L_{0}}\right)^{1 / n}, \\
K=\frac{4 R^{2} \phi^{3}}{72 C(1-\phi)^{2}}, \\
\mu_{\mathrm{eff}}=\mu\left(\frac{3 n+1}{4 n}\right)^{n}\left(\frac{K \phi}{\beta^{2}}\right)^{(1-n) / 2},
\end{gathered}
$$

where $\beta$ and $C$ are empirical constants in the macroscopic model, $R$ is the particle radius, and $\mu_{\text {eff }}$ is the effective viscosity of power-law fluid.

According to (23), (25), and (26), the effective viscosity can be found:

$$
\begin{aligned}
\mu_{\mathrm{eff}}= & \frac{\mu\left[(3+1 / n)\left(3+\left(D_{T} / n\right)-D_{f}\right)\right]^{n}}{4\left(3+D_{T}-D_{f}\right)} \\
& \times\left\{\frac{r_{\max }^{D_{T}} \phi\left(3-D_{T}-D_{f}\right) 2^{D_{T}-1}}{L_{0}^{D_{T}-1}\left[1-\left(r_{\min } / r_{\max }\right)^{3-D_{T}-D_{f}}\right]}\right\}^{1-n} .
\end{aligned}
$$

It can be seen that macroscopic models (27) and (28) have empirical constants, which is not related to the microstructural parameters of a porous medium, whereas there is no empirical constant in the present fractal models (25) and (29), and effects of structural parameters of porous media on the permeability and the effective viscosity of power-law fluids are taken into account in the proposed models.

The shear stress at wall is [19]

$$
\tau_{w}=\frac{r}{2} \frac{\Delta p}{L_{t}}=\frac{r^{D_{T}} \Delta p}{2^{2-D_{T}} L_{0}^{D_{T}}} .
$$


The total shear stress at all walls can be found from

$$
\begin{aligned}
\tau & =-\int_{r_{\min }}^{r_{\max }} \tau_{w} d N \\
& =\frac{D_{f} r_{\max }^{D_{T}}\left[1-\left(r_{\min } / r_{\max }\right)^{D_{T}-D_{f}}\right] \Delta p}{2^{2-D_{T}} L_{0}^{D_{T}}\left(D_{T}-D_{f}\right)} .
\end{aligned}
$$

According to (1) and (31), we can obtain the fluid apparent viscosity $\mu_{a}$ :

$$
\mu_{a}=\frac{\tau}{\dot{\gamma}}=\mu^{1 / n}\left\{\frac{D_{f} r_{\max }^{D_{T}}\left[1-\left(r_{\min } / r_{\max }\right)^{D_{T}-D_{f}}\right] \Delta p}{2^{2-D_{T}} L_{0}^{D_{T}}\left(D_{T}-D_{f}\right)}\right\}^{1-1 / n} .
$$

For non-Newtonian, the apparent viscosity $\mu_{a}$ and effective permeability $K_{e}$ are incorporated in the generalized Darcy law [20]:

$$
V=\frac{K_{e}}{\mu_{a}} \frac{\Delta p}{L_{0}} .
$$

According to (23), (32), and (33), the effective permeability for power-law fluid is obtained:

$$
\begin{aligned}
K_{e}= & \frac{n \phi r_{\max }^{2 D_{T}} 2^{2 D_{T}-3}\left(3-D_{T}-D_{f}\right) L_{0}^{2-2 D_{T}}}{(3 n+1)\left(3+\left(D_{T} / n\right)-D_{f}\right)\left[1-\left(r_{\min } / r_{\max }\right)^{3-D_{T}-D_{f}}\right]} \\
& \times\left[\frac{1-\left(r_{\min } / r_{\max }\right)^{D_{T}-D_{f}}}{D_{T} / D_{f}-1}\right]^{1-1 / n} .
\end{aligned}
$$

It is seen from (19), (23), (29), (32), and (34) that the flow rate, flow velocity, effective viscosity, apparent viscosity, and effective permeability for power-law fluid in porous media are related not only to the structural parameters of porous media but also to fluid characteristic parameters.

\section{Results and Discussion}

The algorithm for determination of the flow velocity and effective permeability for power-law fluid flow in fractal porous media are summarized as follows:

(1) Porosity $\phi$ and the average particle radius $R$ are given $(\phi=0.38, R=0.05 \mathrm{~cm}[18])$.

(2) Find the microstructure parameters $\left(r_{\min }, r_{\max }, L_{0}\right)$ and the fractal dimension $\left(D_{f}, D_{T}\right)$ from (8), (10)(15).

(3) The fluid property parameters $(\mu, n)$ can be obtained by experiment. $\left(\mu=0.2 \mathrm{~Pa} \cdot \mathrm{s}^{n}, n=0.3[18]\right)$.

(4) Find the average flow velocity from (23) and find the effective permeability from (34).

Figure 1 shows a comparison between the fractal flow rate model $(17)\left(r=0.107 \mathrm{~cm}, L_{0}=0.657 \mathrm{~cm}\right.$, and $\left.D_{T}=1.122\right)$

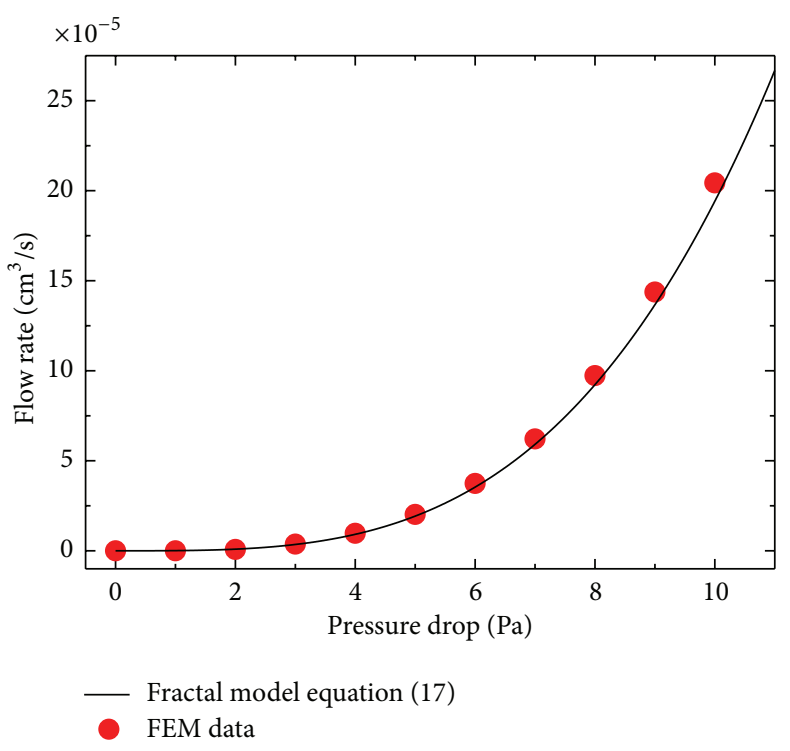

FIGURE 1: The flow rate versus pressure drop between the present fractal model and FEM data.

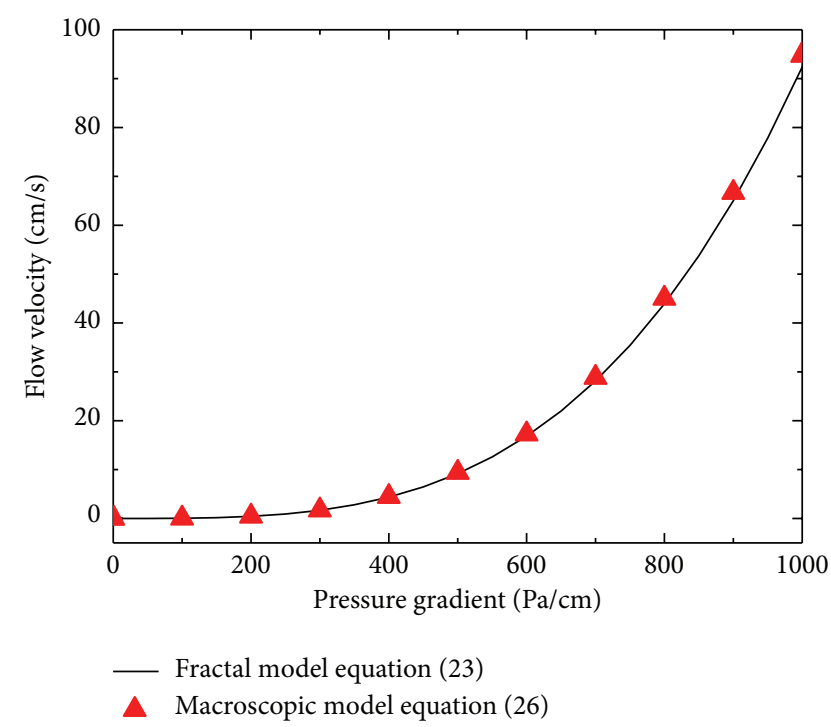

FIgURE 2: The flow velocity versus pressure gradient between the present fractal model and macroscopic model.

and FEM (finite element method) simulation data [18] for power-law fluid with rheological properties $\mu=1.0 \mathrm{~Pa} \cdot \mathrm{s}^{n}$ and $n=0.3$. Good agreement is found between them; therefore, the present fractal capillary model can be used to model power-law fluid flow in a single capillary.

Figure 2 shows a comparison between the fractal velocity model and macroscopic model [18] for power-law fluid flow in packed beds. The parameters for power-law fluid are $\mu=$ $0.2 \mathrm{~Pa} \cdot \mathrm{s}^{n}$ and $n=0.3$, and the parameters for packed beds are $R=0.05 \mathrm{~cm}, \phi=0.38$. In $(26), K=8.135 \times 10^{-6} \mathrm{~cm}^{2}$ and $\mu_{\text {eff }}$ is calculated by (28). The fractal model shows good agreement with macroscopic model. 


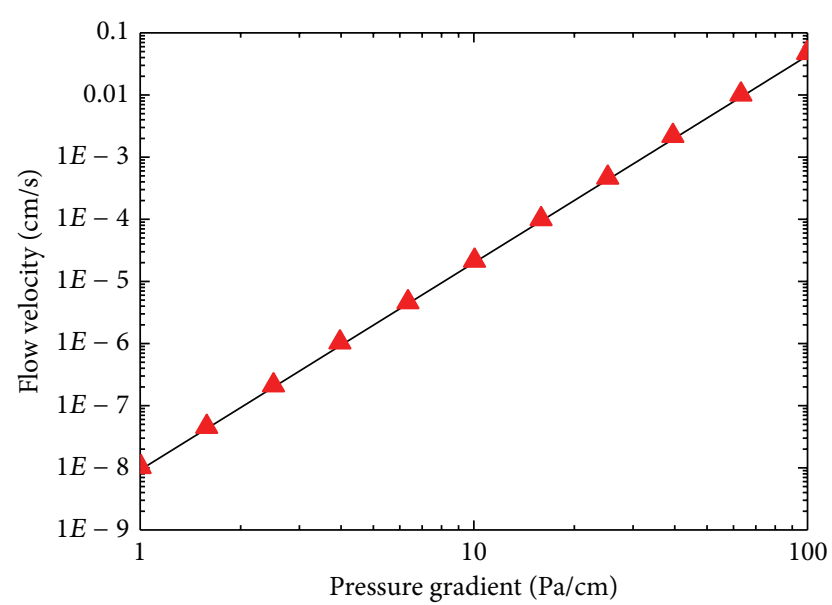

_ Fractal model equation (23)

- Network model data

FIGURE 3: The flow velocity versus pressure gradient between the present fractal model and network model simulation data.

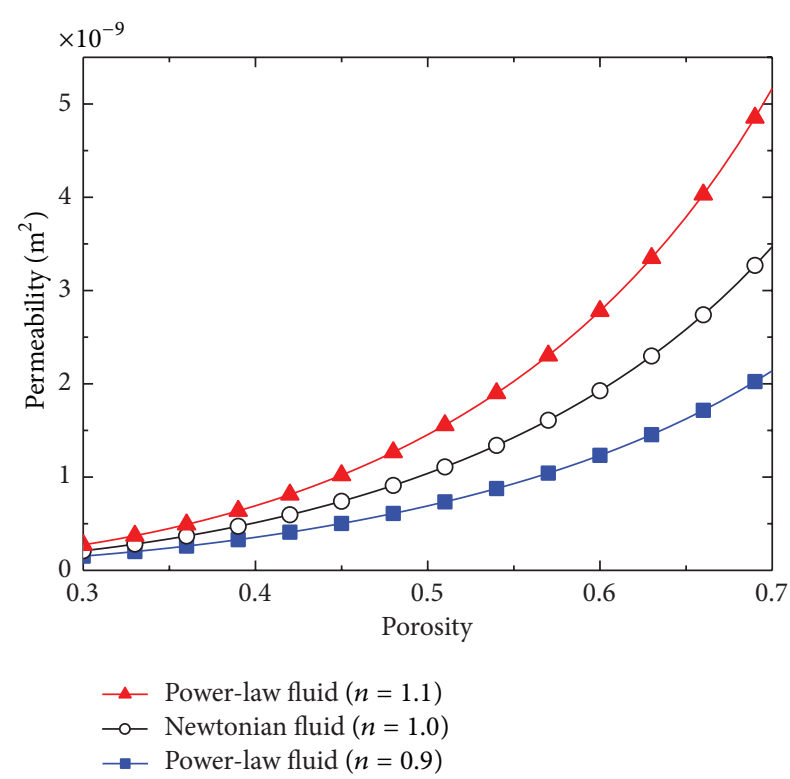

FIGURE 4: The effective permeability (34) versus porosity for powerlaw fluid with different power indexes.

Figure 3 shows a comparison between the fractal velocity model and network model simulation data [18] for power-law fluid with $\mu=0.2 \mathrm{~Pa} \cdot \mathrm{s}^{n}$ and $n=0.3$. The simulation was run for a network generated from a packed bed of $\phi=0.38$ and $R=0.05 \mathrm{~cm}$. There is good agreement between the present fractal model prediction and network model data.

Figure 4 shows a comparison of the effective permeability (34) versus porosity at different power indexes. It can be seen that the effective permeability increases with the increases of porosity and power indexes. This is consistent with the actual situations.

\section{Conclusions}

In conclusion, fractal models for flow rate, velocity, effective viscosity, apparent viscosity, and effective permeability of power-law fluid flow in porous media have been derived based on the fractal geometry theory. The proposed fractal models relate the properties of power-law fluid to the structural parameters of porous media. Good agreement between the fractal models and related data verifies the validity of the present models. The effective permeability increases with the increases of porosity and power indexes. The analytical expressions will contribute to the revealing of the physical principles for the power-law fluids flow in porous media.

\section{Conflict of Interests}

The author declares that there is no conflict of interests regarding the publication of this paper.

\section{Acknowledgments}

This work was supported by the National Natural Science Foundation of China (Grant no. 41202094), the Foundation of State Key Laboratory of Petroleum Resources and Prospecting in China University of Petroleum (Grant no. PRP/open1206), and Open Fund of State Key Laboratory of Oil and Gas Reservoir Geology and Exploitation (Grant no. PLN1113).

\section{References}

[1] G. W. Govier and K. Aziz, The Flow of Complex Mixtures in Pipes, Van Nostrand Reinhold, New York, NY, USA, 1972.

[2] A. J. Katz and A. H. Thompson, "Fractal sandstone pores: implications for conductivity and pore formation," Physical Review Letters, vol. 54, no. 12, pp. 1325-1328, 1985.

[3] H. Nguyen, B. Chopard, and S. Stoll, "Hydrodynamic properties and permeability of fractal objects," International Journal of Modern Physics C, vol. 18, no. 4, pp. 732-738, 2007.

[4] M. J. Yun, W. Zheng, Y. B. Li, and Y. Li, "Fractal analysis of Herschel-Bulkley fluid flow in a capillary," Acta Physica Sinica, vol. 61, no. 16, Article ID 164701, 2012.

[5] M. J. Yun and W. Zheng, "Fractal analysis of Robertson-Stiff fluid flow in porous media," Chinese Physics Letters, vol. 29, no. 6, Article ID 064706, 2012.

[6] B. M. Yu, "Ractal character for tortuous streamtubes in porous media," Chinese Physics Letters, vol. 22, no. 1, pp. 158-160, 2005.

[7] B. M. Yu and P. Cheng, "A fractal permeability model for bidispersed porous media," International Journal of Heat and Mass Transfer, vol. 45, no. 14, pp. 2983-2993, 2002.

[8] B. M. Yu, L. J. Lee, and H. Q. Cao, "A fractal in-plane permeability model for fabrics," Polymer Composites, vol. 23, no. 2, pp. 201-221, 2002.

[9] C. Ö. Karacan and P. M. Halleck, "A fractal model for predicting permeability around perforation tunnels using size distribution of fragmented grains," Journal of Petroleum Science and Engineering, vol. 40, no. 3-4, pp. 159-176, 2003.

[10] B. Q. Xiao, G. P. Jiang, and L. X. Chen, "A fractal study for nucleate pool boiling heat transfer of nanofluids," Science China Physics, Mechanics and Astronomy, vol. 53, no. 1, pp. 30-37, 2010. 
[11] B. Q. Xiao, J. T. Fan, G. P. Jiang, and L. X. Chen, "Analysis of convection heat transfer mechanism in nanofluids," Acta Physica Sinica, vol. 61, no. 15, Article ID 154401, 2012.

[12] J. C. Cai, B. M. Yu, M. Q. Zou, and L. Luo, "Fractal characterization of spontaneous co-current imbibition in porous media," Energy and Fuels, vol. 24, no. 3, pp. 1860-1867, 2010.

[13] J. C. Cai, B. M. Yu, M. Q. Zou, and M. Mei, "Fractal analysis of invasion depth of extraneous fluids in porous media," Chemical Engineering Science, vol. 65, no. 18, pp. 5178-5186, 2010.

[14] J. C. Cai and B. M. Yu, "A discussion of the effect of tortuosity on the capillary imbibition in porous media," Transport in Porous Media, vol. 89, no. 2, pp. 251-263, 2011.

[15] J. C. Cai, X. Y. Hu, D. C. Standnes, and L. You, "An analytical model for spontaneous imbibition in fractal porous media including gravity," Colloids and Surfaces A, vol. 414, pp. 228-233, 2012.

[16] J. C. Cai, L. J. You, X. Y. Hu, J. Wang, and R. Peng, "Prediction of effective permeability in porous media based on spontaneous imbibition effect," International Journal of Modern Physics C, vol. 23, no. 7, Article ID 1250054, 12 pages, 2012.

[17] J. C. Cai and S. Y. Sun, "Fractal analysis of fracture increasing spontaneous imbibition in porous media with gas-saturated," International Journal of Modern Physics C, vol. 24, no. 8, Article ID 1350056, 13 pages, 2013.

[18] M. T. Balhoff and K. E. Thompson, "A macroscopic model for shear-thinning flow in packed beds based on network modeling," Chemical Engineering Science, vol. 61, no. 2, pp. 698719, 2006.

[19] O. N. Cavatorta and R. D. Tonini, "Dimensionless velocity profiles and parameter maps for non-Newtonian fluids," International Communications in Heat and Mass Transfer, vol. 14, no. 4, pp. 359-369, 1987.

[20] C. D. Tsakiroglou, "A methodology for the derivation of nonDarcian models for the flow of generalized Newtonian fluids in porous media," Journal of Non-Newtonian Fluid Mechanics, vol. 105, no. 2-3, pp. 79-110, 2002. 


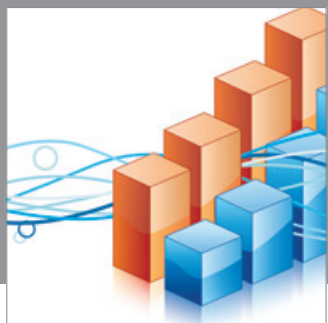

Advances in

Operations Research

mansans

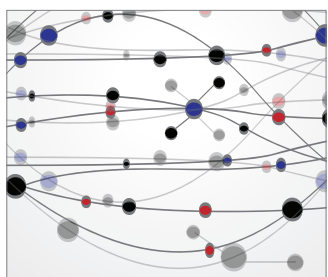

The Scientific World Journal
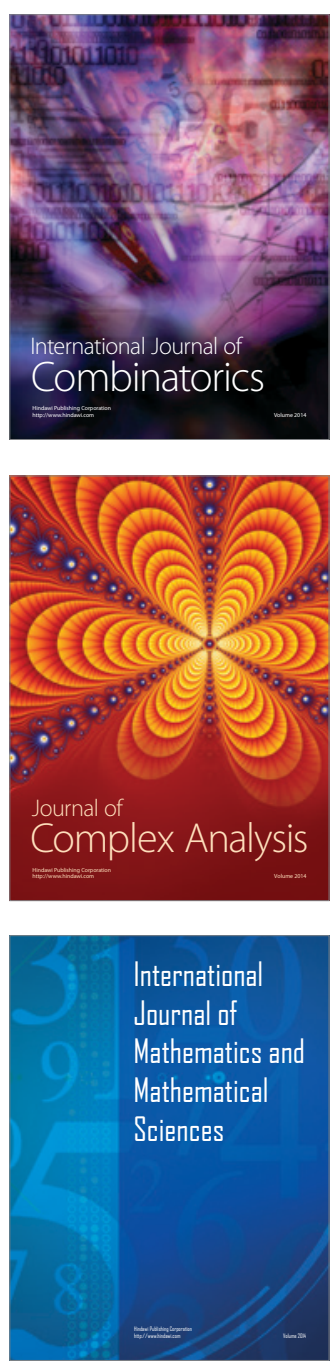
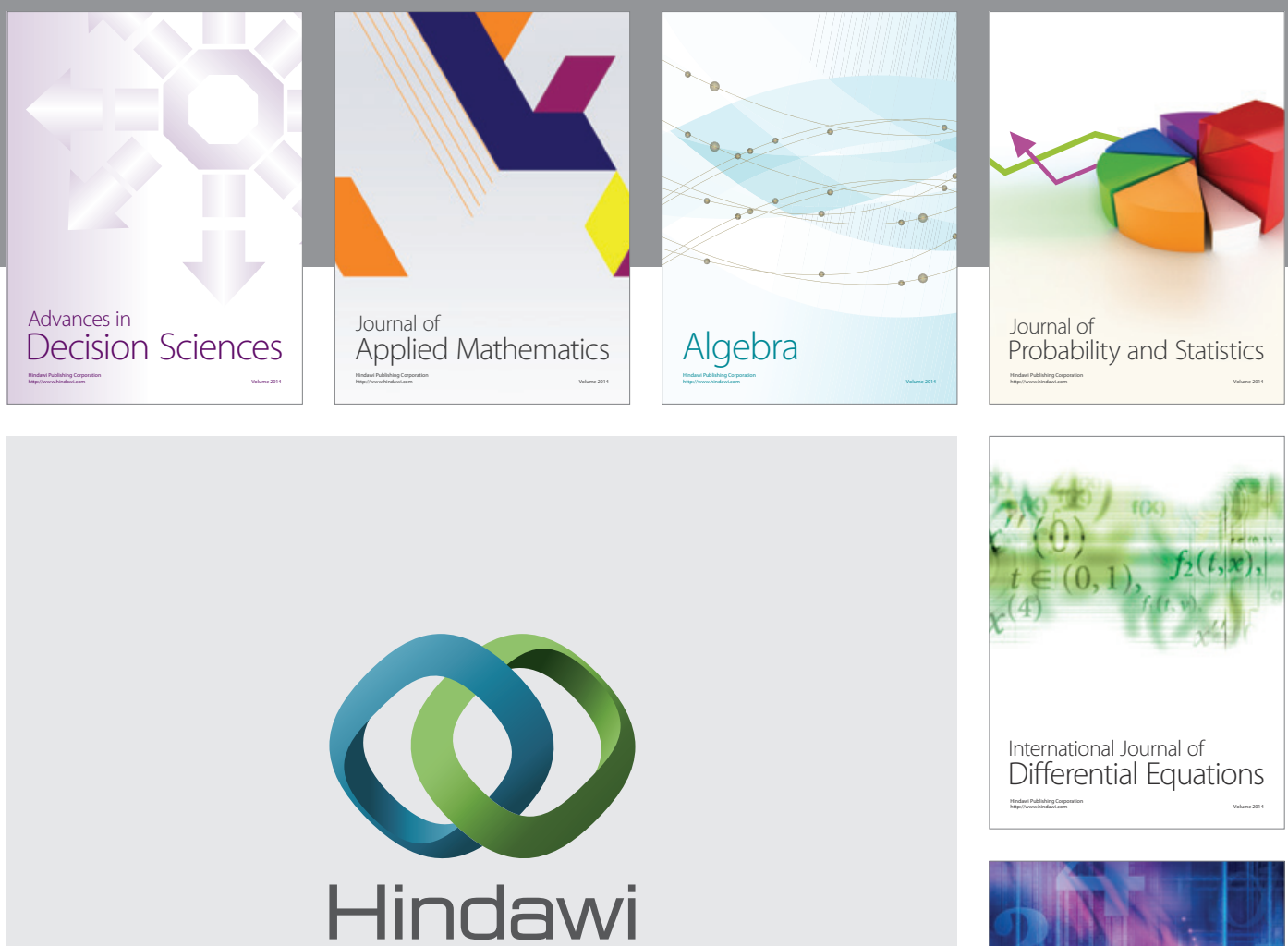

Submit your manuscripts at http://www.hindawi.com
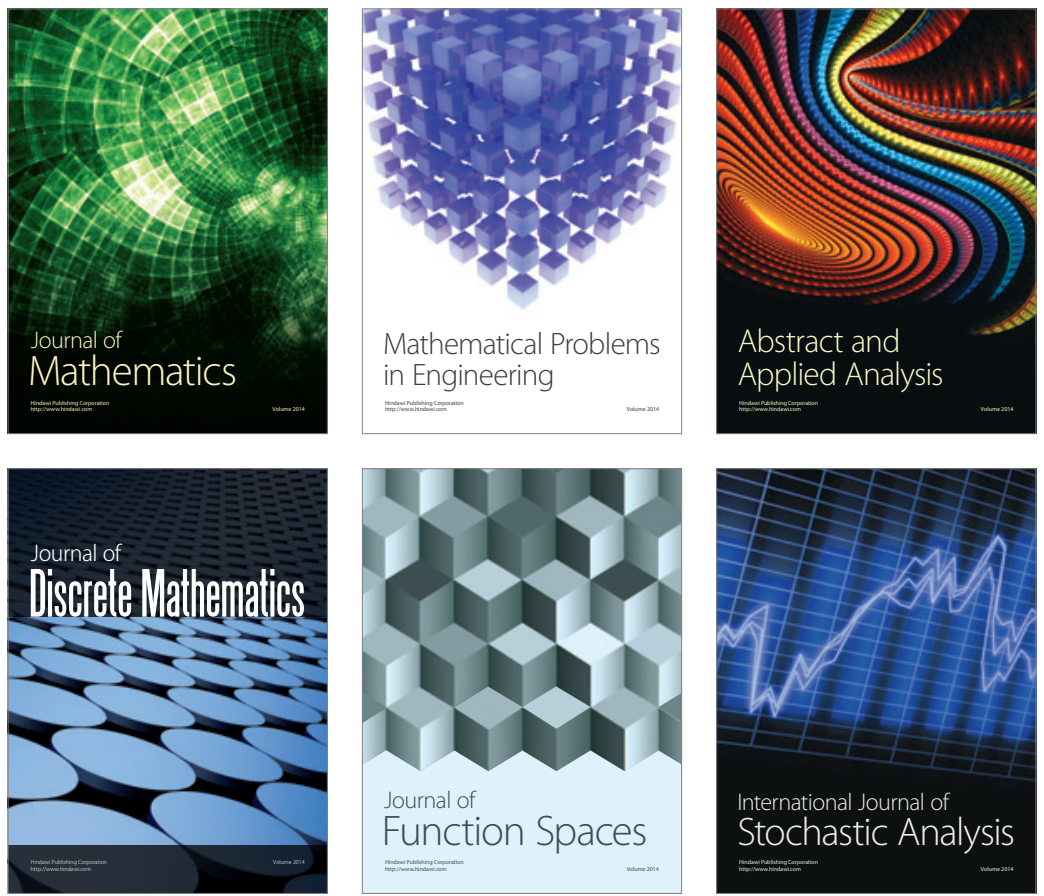

Journal of

Function Spaces

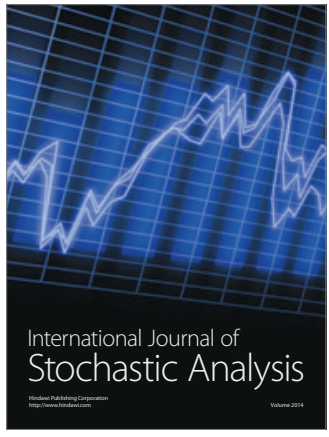

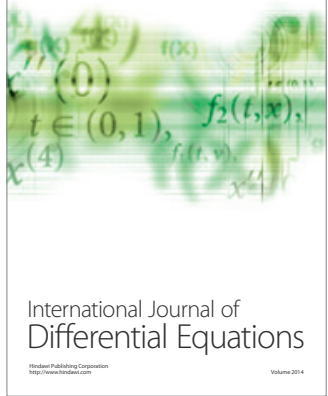
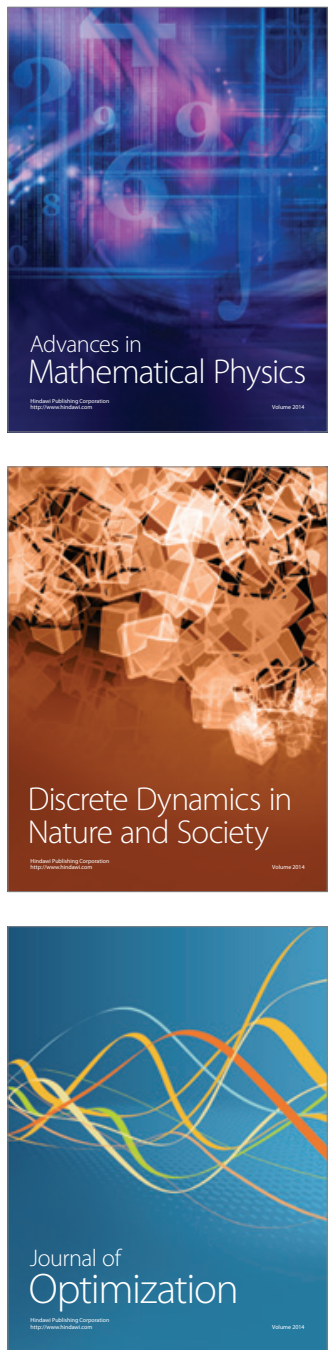